\title{
Consensus on Climate Trends in Western North Pacific Tropical Cyclones
}

\author{
NAM-YOUng KANG AND JAMES B. ElSNER \\ The Florida State University, Tallahassee, Florida
}

(Manuscript received 16 December 2011, in final form 6 May 2012)

\begin{abstract}
Research on trends in western North Pacific tropical cyclone (TC) activity is limited by problems associated with different wind speed conversions used by the various meteorological agencies. This paper uses a quantile method to effectively overcome this conversion problem. Following the assumption that the intensity ranks of TCs are the same among agencies, quantiles at the same probability level in different data sources are regarded as having the same wind speed level. Tropical cyclone data from the Joint Typhoon Warning Center (JTWC) and Japan Meteorological Agency (JMA) are chosen for research and comparison. Trends are diagnosed for the upper $45 \%$ of the strongest TCs annually. The $27-\mathrm{yr}$ period beginning with 1984 , when the JMA began using the Dvorak (1982) technique, is determined to be the most reliable for achieving consensus among the two agencies regarding these trends. The start year is a compromise between including as many years in the data as possible, but not so many that the period includes observations that result in inconsistent trend estimates. The consensus of TC trends between the two agencies over the period is interpreted as fewer but stronger events since 1984, even with the lower power dissipation index (PDI) in the western North Pacific in recent years.
\end{abstract}

\section{Introduction}

The issue of global warming and climate change extends to how tropical cyclone (TC) climatology might be affected. The western North Pacific is the world's most prolific TC basin, accounting for about one-third of total global TC occurrences (Chan 2005). Nevertheless, previous research on $\mathrm{TC}$ trends in this region does not suggest a clear consensus on what is happening. Such inconclusive diagnostics retard further speculation on the TC climate in this basin.

The problem largely stems from discrepancies among the different sources of observations. Webster et al. (2005) analyzed the Joint Typhoon Warning Center (JTWC) best-track data and found increasing trends in the number and proportion of the strongest typhoons. From the same data source, Emanuel (2005) focused on the increase of the power dissipation index (PDI) representing the variability of TC energy and destructiveness and its significant relationship with the rising trend in sea surface temperature. On the other hand, research on TC trends using data from sources such as the Japan Meteorological

Corresponding author address: Nam-Young Kang, Bellamy Building 320, The Florida State University, Tallahassee, FL 32306.

E-mail: nkang@fsu.edu
Agency (JMA) and the Hong Kong Observatory (HKO) show no such increases (Wu et al. 2006; Yeung 2006). Subsequent studies confirmed these inconsistencies (Kamahori et al. 2006; Yu et al. 2007).

These inconsistent diagnostics are a consequence of at least two factors. One factor is the use of different time periods over which the maximum sustained wind (MSW) speed is calculated. For instance, the JTWC uses a 1-min averaging period while the JMA uses a 10-min period. On average, the 1-min wind speeds are about $12 \%$ higher than the 10-min speeds (Atkinson 1974). The other factor is more complicated as it involves the entire procedure by which MSW is estimated using various observational platforms, algorithms, operational procedures, and so on, all of which get modified over time. For historical reasons the different agencies often used different observational platforms, resulting in different wind speed estimates even for the same TC event. Satellites are now the common platform and the Dvorak technique applied to satellite imagery is the routine basis for estimating MSW at most agencies (Levinson et al. 2010).

However, even today there remain possible discrepancies based on differing qualitative processes in how the technique is applied. As an example, there is a decision associated with whether the cloud pattern is a TC or not. Atypical cloud patterns are likely to result in 
inconsistent decisions among the agencies. Indeed, the entire operational practice can be understood as a qualitative best guess and this can lead to inconsistencies in estimates of MSW. Nakazawa and Hoshino (2009) support this idea explaining that JTWC is likely to have faster intensification rates for TCs before maturity and slower weakening rates after maturity relative to the JMA.

In an attempt to compare, Song et al. (2010) and Knapp and Kruk (2010) tried to find a reasonable conversion relationship. They selected coincident TC cases from the different data sources and then applied a statistical model to the data from these cases. Song et al. suggested a nonlinear regression, while Knapp and Kruk retraced the JMA procedure and retrieved 1-min MSWs using the JTWC procedure. These investigations provided clear understanding of interagency differences.

Nonetheless, to continue progress additional things need to be considered. First, finding coincident cases is not trivial, especially for the older datasets. A researcher willing to examine the TC trends will spend time and resources in finding such cases. Second, trends based only on the coincident cases may not represent the true trends owing to the nonrandom selection of cases. Third, fixing one conversion relationship for all years is probably not reasonable as there are nonuniform improvements across agencies. For example, independently launched meteorological satellites and subsequently improved observation procedures would have made any preceding conversion rate no longer suitable for the relationship. Third, the question about reliable trends in western North Pacific TC activity remains unanswered.

The present paper is motivated by these issues. In particular, we suggest using quantiles as an effective solution to overcome the wind speed conversion problem. Since the individual TC intensity can be represented as a lifetime-maximum wind (LMW) speed, quantiles are applied to the set of annual LMWs. The assumption is that intensity ranks of TC events are the same across agencies even if the LMWs differ. This is reasonable given that the different agencies are obtaining intensity estimates from essentially the same set of TCs. The important insight is that from a quantile point of view, the key factor is proportion, not the intensity itself. This convenience makes it possible to compare different observational estimates with the same framework. Elsner et al. (2008) show that a quantile analysis enables intercomparison of TC intensity trends even across different basins. Different observations in the same basins are expected to return the same trends as long as the intensity values adhere to this assumption. Conversion problems will not have an impact when using a quantile approach.

The methodology is based on the empirical framework developed in Kang and Elsner (2012), where TC climatology is examined using four indices including frequency, intensity, activity, and intensity efficiency. We demonstrate the methodology using data from the JWTC and JMA. The results show that a consensus of TC climatology in the western North Pacific is possible when using these two datasets. In particular, a consensus on the $\mathrm{TC}$ trends is found.

The paper is outlined as follows. Data and the research domain are described in section 2. The quantile method for comparing LMW from the JTWC and the JMA is presented in section 3. The method is applied to TC climate indicators in section 4. Diagnostics from direct and quantile comparisons of the TC climate indictors using the two data sources are shown. Based on the comparisons a reliable consensus is demonstrated. Results and conclusions are summarized in section 5. All computations and figures are done using $\mathrm{R}$ (http://www.r-project. org). The code is available from the lead author.

\section{Research data}

\section{a. Data and TC selection}

We focus exclusively on TCs over the western North Pacific basin. In particular, we compare TC climate indices using data from the JTWC (available at www.usno. navy.mil/NOOC/nmfc-ph/RSS/jtwc/best_tracks) and the JMA (available at www.jma.go.jp/jma/jma-eng/jma-center/ rsmc-hp-pub-eg). The JMA has been entitled the Regional Specialized Meteorological Center (RSMC) by the World Meteorological Organization, while JTWC has the longest history of observations in this basin. The geographic domain is $3^{\circ}-35^{\circ} \mathrm{N}, 104^{\circ}-175^{\circ} \mathrm{E}$. The eastern and northern boundaries are set to minimize the influences of interbasin TCs and the midlatitude environment, respectively, and the southern and western boundaries represent statistical and geographical limits, respectively. We select TCs whose genesis and LMW locations are within this domain. Where the LMW location spans more than a single observation, its location is placed at the average latitude and longitude. JMA provide MSW estimates beginning in 1977, so we extract LMW values from both datasets over the 34-yr period 1977-2010. Prior to 1977 , only central pressure values are available for TC intensity in the JMA data. See Kang and Elsner (2012) for more details and additional justification.

\section{b. Histograms of LMW}

Frequency distributions of LMW for the set of TCs extracted from both data sources are presented in Fig. 1. The LMW values are given in units of knots $(1 \mathrm{kt}=$ $0.514 \mathrm{~m} \mathrm{~s}^{-1}$ ) because that is how they are recorded in the datasets. The distributions, given in 5-kt bins, both 
(a)

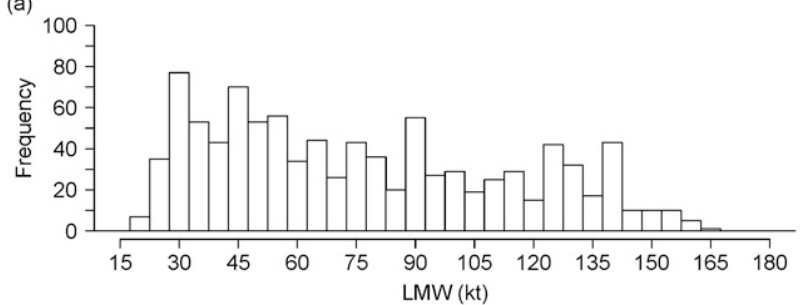

(b)

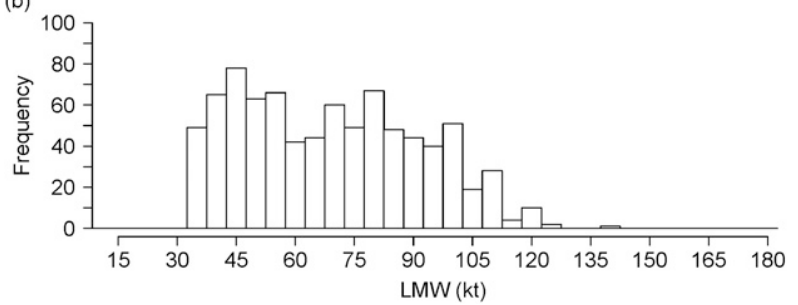

FIG. 1. Histogram of lifetime-maximum wind (LMW) speeds. Data are from (a) JTWC and (b) JMA. Both datasets have wind speeds recorded in knots $(\mathrm{kt})$ at 5 -kt intervals. If a $12 \%$ conversion factor is applied (Atkinson 1974), $140 \mathrm{kt}$ is converted to $157 \mathrm{kt}$ as a 1-min wind speed.

show similar positive skewness (longer right than left tail) but there are obvious differences.

The JTWC data shows a minimum lifetime-maximum wind (LMW) speed of $20 \mathrm{kt}$ whereas the JMA data show a minimum of $35 \mathrm{kt}$. Thus JMA data do not include tropical depressions. Differences at the maximum speeds are also apparent. The JTWC data show a maximum LMW speed of $165 \mathrm{kt}$, whereas the JMA data show a maximum of only $140 \mathrm{kt}$. This difference of $25 \mathrm{kt}$ at the highest intensity wind speeds is not small and suggests nontrivial discrepancies. Following conversion rules, JMA wind speeds can be compared with JTWC values. Among the rules, for example, if a $12 \%$ conversion factor is applied (Atkinson 1974), $140 \mathrm{kt}$ is converted to $157 \mathrm{kt}$ as a 1-min wind speed-still below the $165 \mathrm{kt}$ of JTWC, although much closer.

\section{Quantile method}

The basinwide number of TCs varies from year to year. The count each year depends on a threshold level of intensity. For instance, the number of typhoons in a given year is obtained using a threshold of $33 \mathrm{~m} \mathrm{~s}^{-1}$ for the set of LMW speeds. However, if the LMW for a given TC is recorded with different values depending on the agency, then the time series of annual counts from each agency using the same threshold will likely be dissimilar. Conversely, if two time series of counts are similar even with different threshold LMW speeds, then the corresponding LMWs indicate the same intensity level.

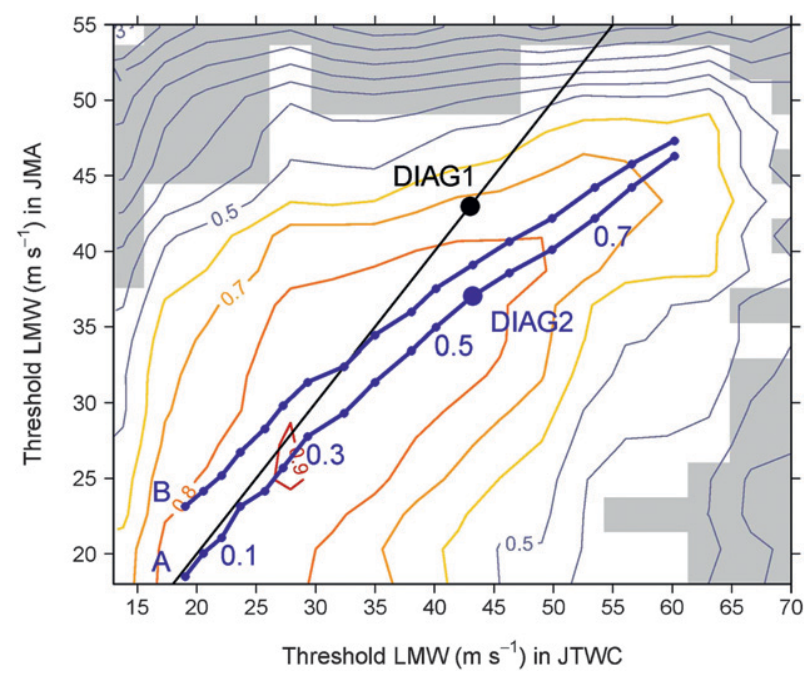

FIG. 2. Contours of correlation between vectors of annual TC numbers from the two agencies at various threshold LMW speeds. Contours are shown at 0.1 intervals. The area of significant correlations at the $5 \%$ level is white. Each significance test uses 1000 paired vectors where each agency's TC counts are randomly sampled with replacement (bootstrapped) from original counts. The diagonal line representing an alignment between the LMW from both agencies is shown in black. Quantile pairs, representing the average values of annual quantiles, are plotted as blue dots in 0.05 quantile intervals and connected by a blue line. Line A represents the case when the lower bound of LMW is set to $17 \mathrm{~m} \mathrm{~s}^{-1}$ for both datasets. Line B represents the case when the lower bound of LMW in the JMA data is modified to $22 \mathrm{~m} \mathrm{~s}^{-1}$ following the intensity tables in Knapp and Kruk (2010). DIAG1 (black circle) indicates $43 \mathrm{~m} \mathrm{~s}^{-1}$, the threshold LMW for a Category 2 storm using the Saffir-Simpson scale (Simpson 1974). DIAG2 (blue circle) indicates the average values of annual quantiles at 0.55 probability level, where the value for JTWC among 16 probability levels is the closest to the threshold in DIAG1.

Therefore, correlating annual count time series at various threshold LMWs will provide a way to match two datasets. Figure 2 is a contour plot of the correlation values over the domains spanned by threshold LMW speeds from each agency using data over the period 1977-2010 (34 yr). The area of significant correlations at the $5 \%$ level is white. Each significance test uses 1000 paired vectors where each agency's TC counts are randomly sampled with replacement (bootstrapped) from original counts. The larger the correlation, the better the match. If the procedures used by both agencies for estimating wind speed are aligned, then the maximum correlation axis runs along the diagonal in this plot. If they are aligned and each produces the same LMW for each TC, then the maximum correlation along the diagonal is one.

Here the results indicate that the procedures are not aligned. Following the assumption that the intensity ranks of events should be the same among agencies although 
LMWs differ in magnitude, an event will likely have the same probability level in both datasets. The quantile is the average of LMW values calculated annually at a given probability level. The 50th percentile ( 0.5 quantile) LMW is $40 \mathrm{~m} \mathrm{~s}^{-1}$ from the JTWC, but only $35 \mathrm{~m} \mathrm{~s}^{-1}$ from the JMA. This bias of stronger TCs in the JTWC dataset relative to the TCs in the JMA dataset is most pronounced for the higher threshold LMW as seen by the slope of the blue lines. The blue lines are computed using 16 probability levels from zero to 0.8 at intervals of 0.05 .

Line A results from the method where we set the lower bounds of LMW in both the JTWC and JMA datasets to $17 \mathrm{~m} \mathrm{~s}^{-1}$. Line B results from the method where we set the lower bound of LMW in the JMA dataset to $22 \mathrm{~m} \mathrm{~s}^{-1}$ following the intensity tables provided in Knapp and Kruk (2010). This table is the mapping table from satellite-derived analysis to TC intensity, which shows comparable 10-min and 1-min wind speeds at the same current intensity (CI) number. Both lines overlaid on the correlation background show similar slopes to that of the correlation ridge line, while line A gives a better correspondence with the ridge especially at lower quantiles. This provides justification for using method A.

The points labeled DIAG1 and DIAG2 on the plot are used for clarifying the quantile method. DIAG1 (black circle) indicates the classic way of direct comparison using the same threshold LMW. This suggests $43 \mathrm{~m} \mathrm{~s}^{-1}$ as the threshold LMW for both JTWC and JMA. In other words, analyses are done for LMWs exceeding $43 \mathrm{~m} \mathrm{~s}^{-1}$, which corresponds to the Saffir-Simpson (Simpson 1974) category 2 and higher. In comparison, DIAG2 (blue circle) reflects the idea of quantile comparison. Here, the JTWC data are taken as a reference. Among 16 probability levels, the quantile at 0.55 probability level is the closest to the threshold LMW of DIAG1. The average quantile at the 0.55 quantile level in JTWC is $43 \mathrm{~m} \mathrm{~s}^{-1}$, which corresponds to $37 \mathrm{~m} \mathrm{~s}^{-1}$ using the JMA data.

\section{Consensus on TC climate trends}

\section{a. TC climate indicators}

Here we follow the definitions of TC climate indicators described in Kang and Elsner (2012), which include frequency (FRQ), intensity (INT), activity (ACT), and the efficiency of intensity (EINT). FRQ is the annual number of TC occurrences whose LMW speeds exceed a threshold level. INT is the annual mean of individual LMW speeds that exceed threshold level. FRQ and INT are calculated for each year and both time series are represented as an ordered vector of values. INT and FRQ are independent since we get INT by dividing the total sum of LMW by FRQ.
ACT is TC activity obtained by combining FRQ and INT at the same time. ACT is computed as

$$
\mathrm{ACT}=[\operatorname{scale}(\mathrm{INT})+\operatorname{scale}(\mathrm{FRQ})] / \sqrt{2},
$$

where the function "scale ( $\cdots)$ " returns standardized vector values. ACT is a linear combination of standardized values of INT and FRQ, indicating the degree to which variations in FRQ and INT are in phase. By definition ACT is an eigenvector from a principal component analysis using FRQ and INT.

Similarly EINT is computed as

$$
\mathrm{EINT}=[\operatorname{scale}(\mathrm{INT})-\operatorname{scale}(\mathrm{FRQ})] / \sqrt{2} .
$$

Opposite from ACT, EINT indicates the degree to which variations in FRQ and INT are out of phase. EINT explains the portion of INT in ACT. Large EINT means that fewer but stronger TCs occur with the same amount of TC energy and vice versa. By definition EINT is the other eigenvector from a principal component analysis using FRQ and INT. As ACT and EINT are the two orthogonal modes of principal component analysis, they are independent. When considering that INT and FRQ can be computed as linear combinations of ACT and EINT, the relationship among the four indicators becomes clear.

\section{b. Direct comparison}

Direct comparison is the standard. It uses the same threshold LMWs for JTWC and JMA. Here we use $43 \mathrm{~m} \mathrm{~s}^{-1}$ for the threshold LMW. TCs whose LMWs exceed this threshold are of Saffir-Simpson category 2 and higher. To aid comparison annual values of each of the four indicators (FRQ, INT, ACT, and EINT) are separately converted to rank probabilities. The annual ranked probability value is the probability referencing the $30-y r$ climatology (1981-2010). The conversion is made for each indicator using the empirical cumulative density function. Subsequently, trends are computed by ordinary least squares regression on the rank probabilities.

Figure 3 shows the diagnostics using the DIAG1 threshold on all four TC climate indicators. Trends have units of ranked probability per 10 years. The trends are the average rate of change in the ranked probabilities over the range of years considered. For example, 0.2 trend (probability/10 yr) in INT would be interpreted as INT is increasing as much as 0.2 rank probability level every 10 years. Trends are presented for different data ranges. Trends using the JTWC and JMA datasets are shown in blue and red lines, respectively. Transparent shades represent $95 \%$ confidence bands on the trend estimates. The band becomes wider as the number of 
(a)

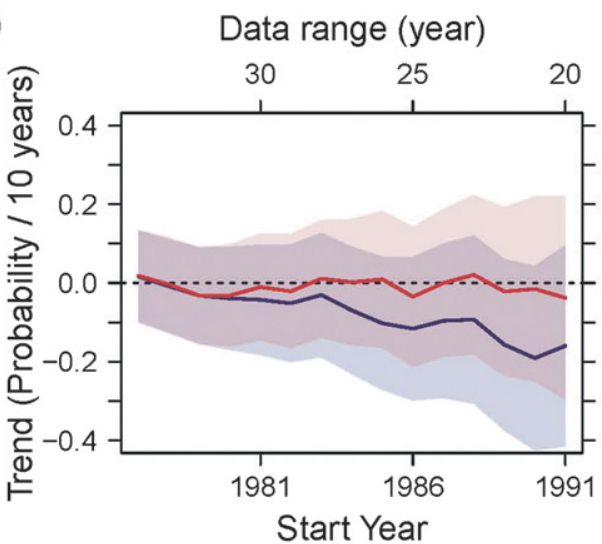

(c)

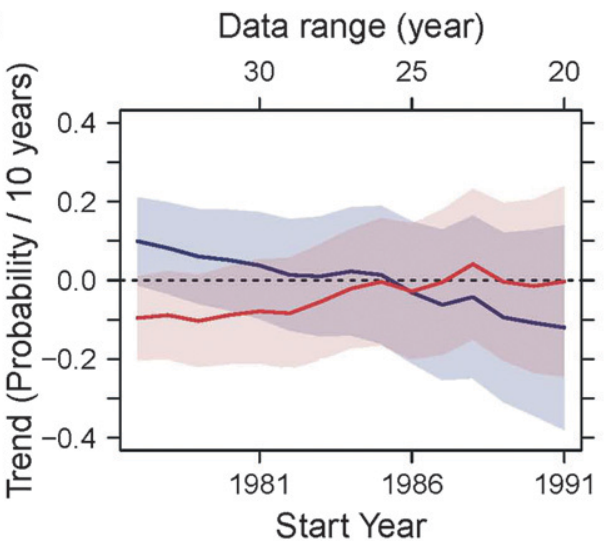

(b)

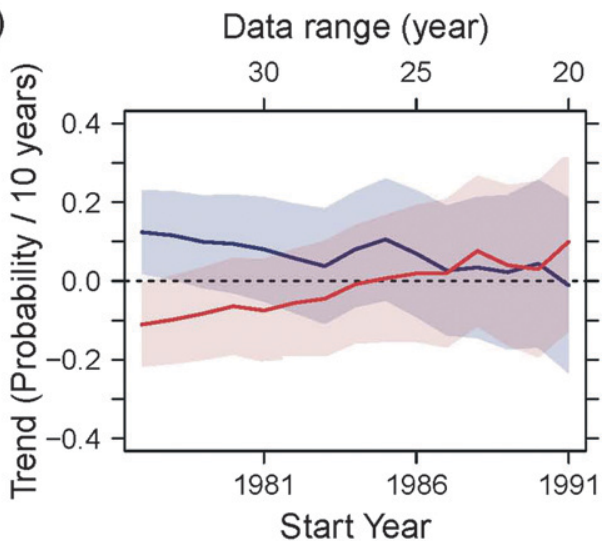

(d)

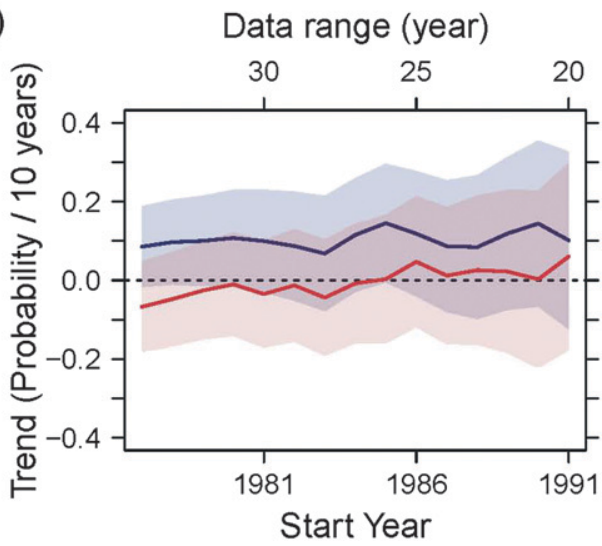

FIG. 3. Tropical cyclone climate trends, computed using the $43 \mathrm{~m} \mathrm{~s}^{-1}$ threshold (see DIAG1 in Fig. 2) as a function of start year. Trends have units of ranked probability per $10 \mathrm{yr}$ and are shown for (a) FRQ, (b) INT, (c) ACT, and (d) EINT. Blue lines are trends based on the collection of TCs in the JTWC dataset, while red lines are for the JMA dataset. Shading in the corresponding color shows the $95 \%$ confidence band. The start year for the trend estimate is shown along the horizontal axis on the bottom and the number of years in the dataset is shown along the horizontal axis at the top.

years in the data sample decreases. The overlap shade (purple) can be thought of as representing a consensus.

Figure 3 clearly demonstrates the issue of inconclusive TC climate trends. The most significant trends appear in INT using the longest (34 yr) data range. Yet it cannot be considered conclusive as the trends have opposite signs (note how they fall on either side of the zero line) using the JTWC and JMA datasets. That is to say, although researchers might find statistically significant increasing trends in INT in the 34-yr (1977-2010) data range using JTWC data, they cannot claim that TCs are getting stronger. The same is true of claims of decreasing trends using the JMA dataset. Note that opposite trends also appear in ACT and EINT by their connection with INT.

The more recent years show no significant trends using either agency's data when considering the $95 \%$ confidence band. The JTWC trends show decreasing FRQ and $\mathrm{ACT}$ and increasing EINT. In contrast, the JMA trends are essentially zero with all TC climate indicators. Fewer TC cases exceeding a relatively high threshold intensity might be the reason for the lack of trends in the JMA data.

\section{c. Quantile comparison}

Researchers using a high value of threshold LMW for trend analysis, as in DIAG1, assume that results represent the tendency of the stronger portion of TCs. That is, the threshold LMW is regarded as an indication of the upper intensity portion (quantile) of stronger TCs. However, the quantile associated with the same LMW threshold may be different between agencies. Or, from the quantile point of view, specific threshold LMW may represent different proportional intensities depending on year. For these reasons the quantile method indicating threshold LMW of specific proportion may be considered a reasonable alternative to the traditional analysis. 
(a)

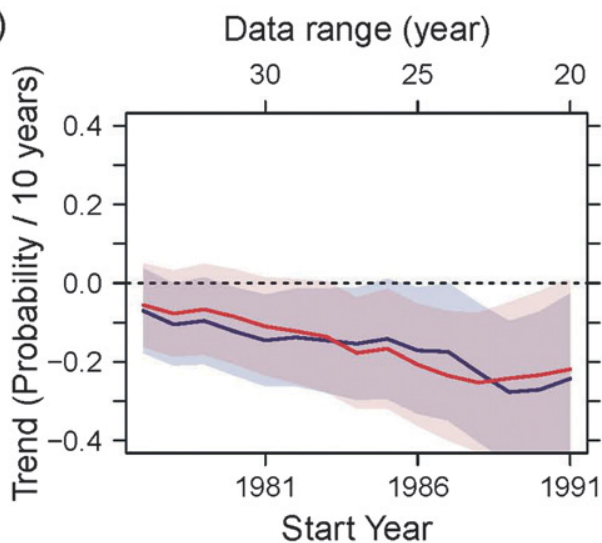

(c)

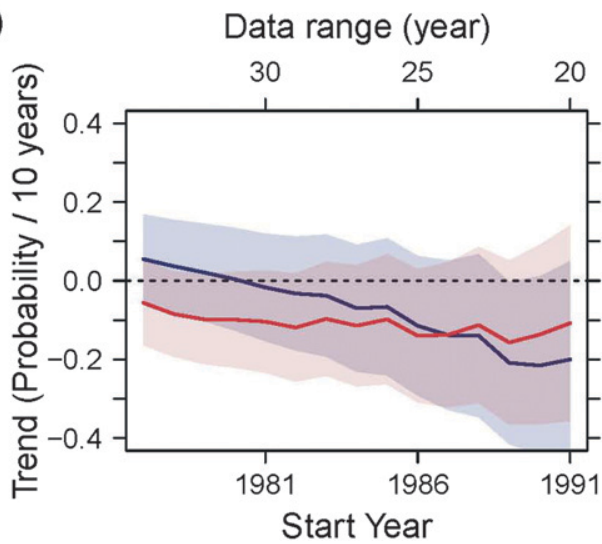

(b)

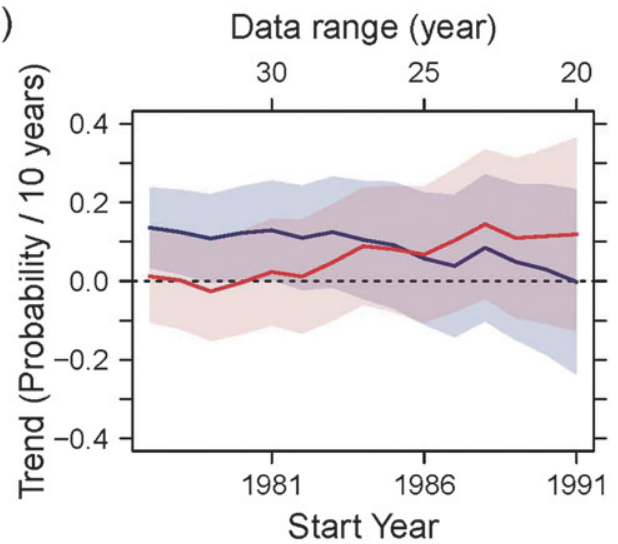

(d)

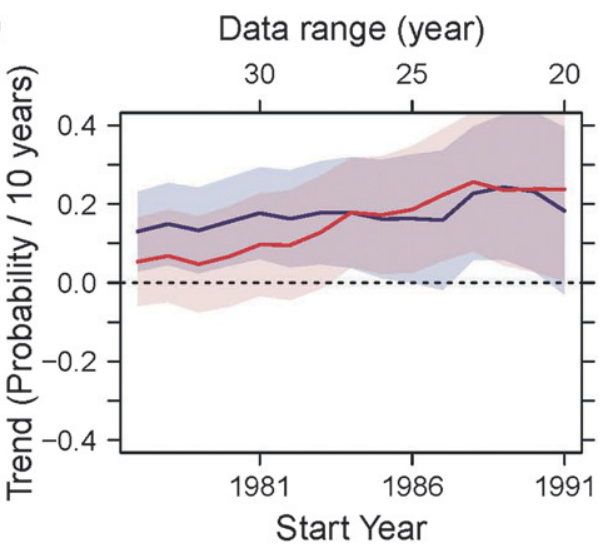

FIG. 4. As in Fig. 3, but the trends are computed using the 0.55 quantile threshold (see DIAG2 in Fig. 2).

Here we consider DIAG2 (see Fig. 2) representing TCs that exceed the 0.55 quantile intensity level. This means that annually only the upper $45 \%$ of the strongest TCs are analyzed. As in the previous section, annual values of ranked probabilities referencing a 30-yr climatology (1981-2010) are used to calculate trends. Results using 0.55 quantile as threshold LMW are shown in Fig. 4. As explained, two different analyses named as DIAG1 and DIAG2 are based on threshold LMW and threshold quantile, respectively. DIAG1 uses a fixed threshold LMW of $43 \mathrm{~m} \mathrm{~s}^{-1}$, while DIAG2 uses the 0.55 quantile as the threshold so that the mean LMW at the quantile is close to $43 \mathrm{~m} \mathrm{~s}^{-1}$. Since annual LMW values at the 0.55 quantile are variant around $43 \mathrm{~m} \mathrm{~s}^{-1}$, analyzed JTWC trends in DIAG2 may not be the same as that in DIAG1, although similar. The results demonstrate a clear improvement in consensus between the JTWC and JMA. As opposed to the earlier results, the trends have the same sign across most data ranges. In addition the trends appear clear.

Despite the general improvements large discrepancies appear in the longest data ranges (1977-2010). It makes sense that better consensus is available using the shorter data ranges since the shorter data ranges include only the most recent data. Improvements in the observational techniques and communication between the agencies might be a few of the reasons. Nevertheless, the discrepancies in INT and ACT trends get larger again in relatively shorter data ranges. This can arise from only a few discrepancies in small samples.

Overall the most reliable consensus of TC trends occurs over data ranges that are not too long or too short. Consensus around a 25-yr range is the most noticeable among the other ranges. Over these ranges, the trends of the upper $45 \%$ of stronger TCs are characterized as decreasing FRQ, increasing INT, decreasing ACT, and increasing EINT. Decreasing FRQ and increasing EINT appear to be the most significant among the trends. Increasing EINT implies that fewer but stronger TCs are occurring, even with the decreasing TC energy represented by ACT.

\section{d. Reliable consensus}

The trends shown in Fig. 4 are computed along four axes including the two observed axes of FRQ and INT and the two principal component axes of ACT and 

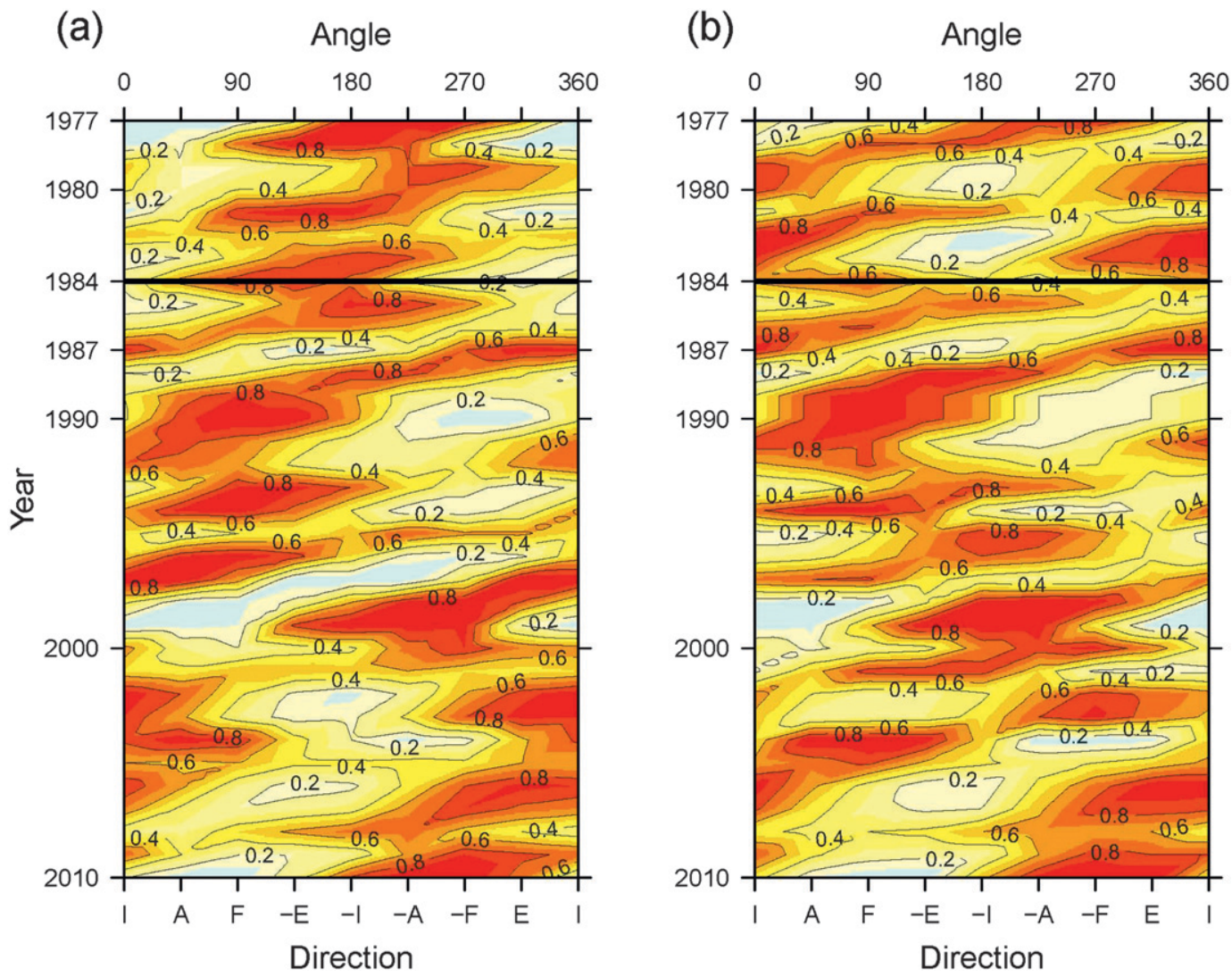

FIG. 5. Annual variations of ranked probabilities in the TC climate indicators using data from the (a) JTWC and (b) JMA: a 0.55 quantile is used as threshold LMW level (see DIAG2 in Fig. 2). Probabilities are computed in $1^{\circ}$ increments around the phase of the plane spanned by the variable and principal component axes composed of FRQ, INT, ACT, and EINT and their opposites. Contours indicate probability levels. Climate indicators are shown along the horizontal axis on the bottom and the equivalent angles are shown along the horizontal axis at the top. The horizontal line at 1984 represents the start year of a reasonable pattern match.

EINT. Since each pair of axes is orthogonal and the two axis pairs are offset by $\pi / 4$, we can examine trends using a single framework. This is done by computing rank probabilities for the upper $45 \%$ of the strongest TCs in off-axes directions and then plotting them in a directionversus-time graph.

Figure 5 shows the results. Years are plotted along the vertical axis and direction is plotted along the horizontal axis where INT, ACT, FRQ, and EINT are denoted as I, $\mathrm{A}, \mathrm{F}$, and $\mathrm{E}$, respectively. The four TC climate indicators are linked in a circular form: .../I/A/F/-E/-I/-A/-F/ $\mathrm{E} / \mathrm{I} / \ldots$. . Negative signs are used to complete the circular framework. Here angles of $0^{\circ}, 45^{\circ}, 90^{\circ}, 135^{\circ}, 180^{\circ}, 225^{\circ}$, $270^{\circ}, 315^{\circ}$, and $360^{\circ}$ indicate $\mathrm{I}, \mathrm{A}, \mathrm{F},-\mathrm{E},-\mathrm{I},-\mathrm{A},-\mathrm{F}, \mathrm{E}$, and I, respectively. Figure 6 shows the relationship between angles and TC climate indicators. Note that $\theta$ is the angle starting from INT. ACT is the case when $\theta$ is $45^{\circ}$. Likewise, EINT is the case when $\theta$ is $315^{\circ}$. ACT or EINT is a variable where equal weights of INT and FRQ are taken into account. Indicators have rank probability values for each year, as shown in Fig. 5. The trends are inferred by examining a vertical line through the plot. For example, the upward trend in EINT, noted in the lower-right panel of Fig. 4, is seen along the $315^{\circ}$ line where the blue and yellow contours give way to red contours as you move down the line from 1977 to 2010.

Separate plots are made using data from the two agencies. A qualitative comparison is made by visually examining the contour patterns between the two plots. The patterns are quite distinct prior to 1984 (horizontal line), but afterward there is good correspondence. The history of observational techniques may help to explain the similarity of TC climatologies between the two agencies. Algorithms using satellite imagery have been largely influenced by Dvorak $(1975,1982,1984)$ techniques. JTWC began to use the Dvorak technique operationally in the 1970s (Chu et al. 2002). JTWC have used the original Dvorak (1975) table for the relationship between CI and MSW. Although the pressurewind relationship from Dvorak (1975) was replaced by 


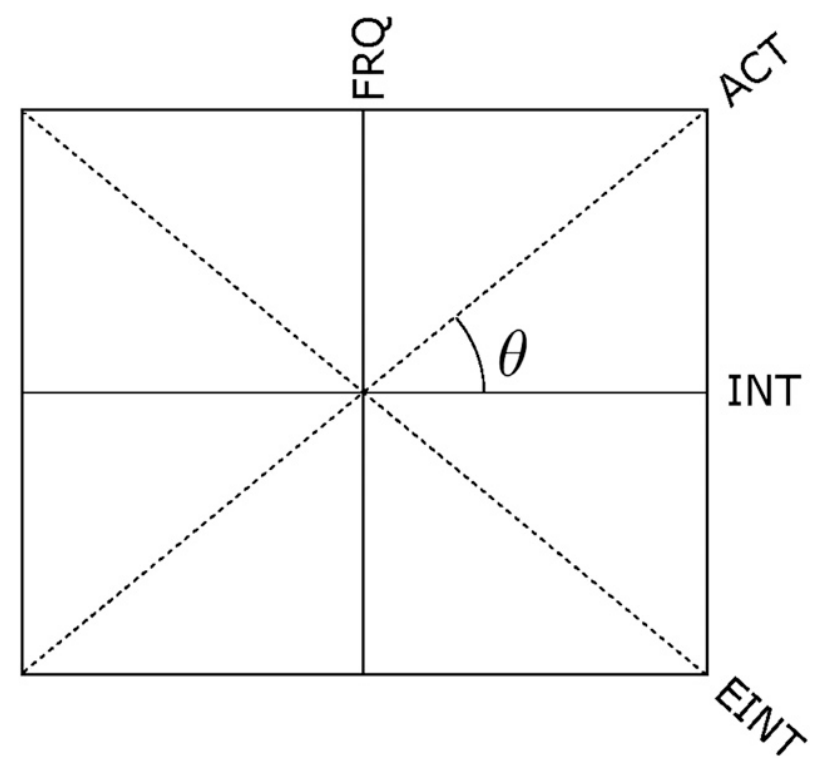

FIG. 6. Schematic of TC climate indicators: $\theta$ is the angle starting from INT; ACT is the case when $\theta$ is $45^{\circ}$. ACT or EINT is a variable where equal weights of INT and FRQ are taken into account. Modified from Kang and Elsner (2012).

Atkinson and Holliday (1977), the CI to maximum sustained wind (MSW) relationship remained unchanged. Using Dvorak (1982), JMA began operational Dvorak analysis in 1984 (see www.wmo.int/pages/prog/www/tcp/ documents/JMAoperationalTCanalysis.pdf). In 1987, JMA introduced the Dvorak (1984) Enhanced Infrared (EIR) technique for intensity analysis. Until August 1987, however, aircraft observation played a major role in TC intensity estimation from both agencies. After 1987, which marks the termination of U.S. Air Force aircraft reconnaissance, satellite observation came to be the major source of information determining TC intensity. Since 1990, JMA operations have referred to the conversion table that assigns Dvorak CI to MSW following Koba et al. (1991).

All of the events mentioned above would have influenced the skill at which the MSW was estimated. Comparison of the two patterns in Fig. 5 seems to indicate that the JMA operational change in 1984 is the most critical. Prior to 1984 , the distribution of ranked probabilities in JMA shows a very different pattern from JTWC. Pattern correlations support the idea. The pattern correlation over the period $1977-83$ is 0.36 , while it shifts to 0.73 over the period 1984-2010. However, we cannot assert that the discrepancy prior to 1984 derives only from JMA observations. For example, Chu et al. (2002) referred to the lower quality of best-track data prior to 1985 in JTWC. As discussed in the previous section, Fig. 5 confirms that the trends in shorter ranges are likely influenced by fewer events. For instance, INT in JTWC shows larger probability values in the late 1990s and the early 2000s. This seems to contribute to the lack of trends noted using the JTWC data when the trends are computed over a smaller number of years.

Results point to the conclusion that the 27-yr period starting with 1984 provides the most reliable consensus on TC climate trends in the western North Pacific. This period gives the longest data range, removing unreliable observations prior to 1984 while achieving robustness against a small sample size. Once we accept this consensus of reliability, the TC climate trends in the western North Pacific are summarized as decreasing FRQ, increasing INT, decreasing ACT, and increasing EINT. The trends can be interpreted as demonstrating that fewer but stronger events are occurring, even with lower PDI.

\section{Conclusions}

This study tries to find a consensus for a period of record over which trends in TC climate in the western North Pacific can be trusted. The study uses observations from the JTWC and the JMA to demonstrate a methodology based on quantiles that is useful for finding such a period. Merits of the quantile approach include the following:

- There is no need to find individual coincident cases among TCs from different agencies. Instead, annual quantiles at various probability levels indicate comparable intensities on a year by year basis.

- All TCs within a chosen spatial domain are used. Accordingly, better diagnostics are available rather than diagnostics based on a limited sample of matched cases.

- Temporal variation in the conversion relationship between agencies is allowed since quantiles were calculated separately for each year.

Using the empirical framework developed in Kang and Elsner (2012), TC climate trends in JTWC and JMA observations are investigated over 34 years (1977-2010). Results provide evidence that a reliable consensus of TC climate trends is available using data over the period 1984-2010. The findings are summarized as follows:

- The quantile method using the upper $45 \%$ of the strongest TCs ( 0.55 quantile as threshold LMW) shows improved consensus between JTWC and JMA compared to the traditional direct method.

- TC climatology between JTWC and JMA shows that the observations prior to 1984 lack reliability and increase the diagnostic discrepancies. 
- Trends in shorter data ranges are likely influenced by fewer events.

- The 27-yr period (1984-2010) is considered as the range that provides the most reliable consensus. That is the longest available data range, removing unreliable observations prior to 1984 and also achieving robustness to small sample sizes.

- Assuming the consensus implies reliable trends, the TC climate in the western North Pacific is summarized by decreasing FRQ, increasing INT, decreasing ACT, and increasing EINT trends.

- Decreasing FRQ and increasing EINT are the most significant.

- The consensus of TC climate trends between the two agencies can be understood as demonstrating that fewer but stronger events are occurring, even with the lower PDI in the western North Pacific in recent years.

Despite the consensus of TC climate trends, there remain a couple of things to consider before accepting them as true. First, improvements in satellite resolution may have caused increased intensity estimates, especially for systems with small eyes. The objective Dvorak technique (ODT) by Velden et al. (1998) represents the improvement of observational technique with highresolution, real-time global digital satellite data and improved computer processing resources. Since the technique works better on stronger TCs with a coherent eye structure (Velden et al. 2006) and higher-resolution imagery enables detecting more TCs with small eyes, the increasing intensity trends might be in part an artifact of this improvement. Intensity trends could be influenced by high-resolution effects even before ODT was employed. On the other hand, we cannot ignore the fact that the termination of aircraft reconnaissance in 1987 could have degraded TC intensity observations. Aircraft reconnaissance in the 1970s and 1980s made a large contribution to finding the structures and characteristics of TCs (Sheets 1990). Although this method contains uncertainties, its direct wind observation greatly helped lessen the uncertainty associated with pattern recognition methods such as the Dvorak technique. The termination of aircraft reconnaissance might have exerted a different influence from the advanced satellite techniques on our trend analysis.

While this study is not the final word on this subject, it demonstrates the utility of the quantile method for solving some problems associated with inhomogeneity in data sources and it provides strong evidence that the data are in reliably good agreement after 1984. Further investigations on the variability of TC climatology, including the portion of the variability related to environmental factors, are now possible. Here we make no attempt to attribute the trends to long-term global climate change as the period of record is simply too short.

Acknowledgments. Partial support for this publication comes from the Geophysical Fluid Dynamics Institute at The Florida State University.

\section{REFERENCES}

Atkinson, G. D., 1974: Investigation of gust factors in tropical cyclones. Joint Typhoon Warning Center Tech. Note JTWC 74-1, 9 pp.

_ , and C. R. Holliday, 1977: Tropical cyclone minimum sea level pressure/maximum sustained wind relationship for the western North Pacific. Mon. Wea. Rev., 105, 421-427.

Chan, J. C. L., 2005: Interannual and interdecadal variations of tropical cyclone activity over the western North Pacific. Meteor. Atmos. Phys., 89, 143-152.

Chu, J. H., C. R. Sampson, A. S. Levine, and E. Fukada, 2002: The Joint Typhoon Warning Center tropical cyclone best tracks, 1945-2000. Joint Typhoon Warning Center, 22 pp.

Dvorak, V. F., 1975: Tropical cyclone intensity analysis and forecasting from satellite imagery. Mon. Wea. Rev., 103, 420-430.

_ 1982: Tropical cyclone intensity analysis and forecasting from satellite visible or enhanced infrared imagery. NOAA National Environmental Satellite Service, Applications Laboratory Training Notes, $42 \mathrm{pp}$.

_ 1984: Tropical cyclone intensity analysis using satellite data. NOAA Tech. Rep. 11, 45 pp.

Elsner, J. B., J. P. Kossin, and T. H. Jagger, 2008: The increasing intensity of the strongest tropical cyclones. Nature, 455, 92-95, doi:10.1038/nature07234.

Emanuel, K. A., 2005: Increasing destructiveness of tropical cyclones over the past 30 years. Nature, 436, 686-688.

Kamahori, H. N., N. Yamazaki, N. Mannoji, and K. Takahashi, 2006: Variability in intense tropical cyclone days in the western North Pacific. SOLA, 2, 104-107.

Kang, N.-Y., and J. B. Elsner, 2012: An empirical framework for tropical cyclone climatology. Climate Dyn., 39, 669-680, doi:10.1007/s00382-011-1231-x.

Knapp, K. R., and M. C. Kruk, 2010: Quantifying interagency differences in tropical cyclone best-track wind speed estimates. Mon. Wea. Rev., 138, 1459-1473.

Koba, H., T. Hagiwara, S. Osano, and S. Akashi, 1991: Relationships between CI number and minimum sea level pressure/ maximum wind speed of tropical cyclones. Geophys. Mag., 44, $15-25$.

Levinson, D. H., H. J. Diamond, K. R. Knapp, M. C. Kruk, and E. J. Gibney, 2010: Toward a homogenous global tropical cyclone best-track dataset. Bull. Amer. Meteor. Soc., 91, 377-380.

Nakazawa, T., and S. Hoshino, 2009: Intercomparison of Dvorak parameters in the tropical cyclone datasets over the western North Pacific. SOLA, 5, 33-36.

Sheets, R. C., 1990: The National Hurricane Center-Past, present, and future. Wea. Forecasting, 5, 185-232.

Simpson, R. H., 1974: The hurricane disaster potential scale. Weatherwise, 27, 169-186.

Song, J. J., J. Wang, and L. Wu, 2010: Trend discrepancies among three best track data sets of western North Pacific 
tropical cyclones. J. Geophys. Res., 115, D12128, doi:10.1029/ 2009JD013058.

Velden, C., T. Olander, and R. Zehr, 1998: Development of an objective scheme to estimate tropical cyclone intensity from digital geostationary satellite imagery. Wea. Forecasting, 13, 172-186.

— , and Coauthors, 2006: The Dvorak tropical cyclone intensity estimation technique: A satellite-based method that has endured for over 30 years. Bull. Amer. Meteor. Soc., 87, 1195-1210.

Webster, P. J., G. J. Holland, J. A. Curry, and H.-R. Chang, 2005: Changes in tropical cyclone number, duration, and intensity in a warming environment. Science, 309, 1844-1846, doi:10.1126/ science. 1116448.

Wu, M. C., K. H. Yeung, and W. L. Chang, 2006: Trends in western North Pacific tropical cyclone intensity. Eos, Trans. Amer. Geophys. Union, 87, 537-538, doi:10.1029/2006EO480001.

Yeung, K. H., 2006: Issues related to global warming-Myths, realities and warnings. Hong Kong Observatory Reprint 647, 16 pp. [Available online at http://www.weather.gov.hk/publica/ reprint/r647.pdf.]

$\mathrm{Yu}, \mathrm{H}$., C. Hu, and L. Jiang, 2007: Comparison of three tropical cyclone intensity datasets. Acta Meteor. Sin., 21, 121-128. 